Equation (4) shows that the remainder of the elements of the first row of $u^{-1}$ can be calculated in order from each succeeding line of the table by performing the operations indicated on columns (3) and (5) respectively. The other elements in the upper righthand corner of $u^{-1}$ then are computed from Eq. (5) which gives for instance when $i=3$ and $j=4$,

$$
\begin{aligned}
10^{6} a_{34} & =10^{6} a_{14}+I_{4} \times 10^{6} / G J_{s} \sum_{n=1}^{n-2} l_{n}=-140.875+1.23396 \times(34+34) \\
& =-56.966 .
\end{aligned}
$$

The inverse matrix now can be completed quickly by filling in the lower left-hand corner according to Eq. (6), so that

$$
u^{-1}=10^{-6} \times\left[\begin{array}{rrrr}
0.0160803 & -2.4179 & -4.7183 & -140.875 \\
0.0160803 & 0.1678 & -2.1326 & -98.920 \\
0.0160803 & 0.1678 & 0.4532 & -56.966 \\
0.0160803 & 0.1678 & 0.4532 & 19.786
\end{array}\right] \text {. }
$$

\title{
ON THE PROBLEM OF HEAT CONDUCTION IN A SEMI-INFINITE RADIATING WIRE*
}

\section{By ARNOLD N. LOWAN (Math. Tables Project, Nat. Bureau of Standards)}

R. V. Churchill derives the solution of the problem of heat conduction in a semiinfinite radiating wire when the initial temperature is zero, and the boundary temperature is a constant. It is the object of this paper to derive the general solution corresponding to an arbitrary initial temperature distribution when the boundary temperature is a prescribed function of time.

Let $k, c, \rho, s, A, h$ and $\alpha=k / \rho c$ denote the thermal conductivity, specific heat, density, perimeter, cross-sectional area, coefficient of heat transfer, and thermal diffusivity of the wire, respectively. Further, let $a=h s / c \rho A$ and $b=a T_{2}$, where $T_{2}$ is the temperature of the medium. If the wire is sufficiently thin so that the temperature may be assumed to be constant over the entire cross section, the problem becomes one-dimensional and the temperature $T(x, t)$ must satisfy the following differential equation, initial and boundary conditions:

$$
\begin{array}{rlrl}
\left(\frac{\partial}{\partial t}-\alpha \frac{\partial^{2}}{\partial x^{2}}+a\right) T(x, t)=b & (x>0, t>0), \\
\lim _{t \rightarrow 0} T(x, t)=f(x), & (2) ; & T(0, t) & =\varphi(t) .
\end{array}
$$

It is easily verified that the expression

$$
T(x, t)=e^{-a t} u(x, t)+v(x, t)
$$

* Received July 17, 1944.

1 R. V. Churchill, Modern operational methods in engineering, McGraw-Hill Book Company, New York, 1944. p. 119. 
satisfies Eqs. (1), (2) and (3), provided the functions $u(x, t)$ and $v(x, t)$ satisfy the following differential equations, initial and boundary conditions:

$$
\begin{gathered}
\frac{\partial u}{\partial t}-\alpha \frac{\partial^{2} u}{\partial x^{2}}=0 \quad(x>0, t>0), \\
\lim _{t \rightarrow 0} u(x, t)=f(x), \quad(6) ; \\
\frac{\partial v}{\partial t}-\alpha \frac{\partial^{2} v}{\partial x^{2}}=-a v+b, \quad(8) ; \quad \lim _{t \rightarrow 0} v(x, t)=0, \quad(9) ; \quad v(0, t)=0 . e^{a t} \varphi(t),
\end{gathered}
$$

From Eqs. (5), (6) and (7), it is clear that $u(x, t)$ is the temperature in a semiinfinite solid initially at the temperature $f(x)$ and with its bounding plane $x=0 \mathrm{kept}$ at the temperature $e^{a t} \varphi(t)$. Using the expression of $u(x, t)$ given by H. S. Carslaw, ${ }^{2}$ we obtain

$$
\begin{aligned}
e^{-a t} u(x, t)= & \frac{e^{-a t}}{2 \sqrt{\pi \alpha t}} \int_{0}^{\infty} f(\xi)\left\{e^{-(x-\xi)^{2} /(4 \alpha t)}-e^{-(x+\xi)^{2} /(4 \alpha t)}\right\} d \xi \\
& +\frac{x}{2 \sqrt{\pi \alpha}} \int_{0}^{t} e^{-a \eta} \varphi(t-\eta) e^{-x^{2} / 4 \alpha \eta} \eta^{-3 / 2} d \eta
\end{aligned}
$$

With the aid of the identity

$$
\int_{0}^{\infty} e^{-\alpha \beta^{2} t} \cos \beta(x-\xi) d \beta=\frac{\sqrt{\pi}}{2 \sqrt{\alpha t}} e^{-(x-\xi)^{2} / 4 \alpha t},
$$

the first term of (11) may be written in the alternative form

$$
\frac{2}{\pi} e^{-a t} \int_{0}^{\infty} \int_{0}^{\infty} e^{-\alpha \beta^{2} t} f(\xi) \sin \beta x \sin \beta \xi d \beta d \xi
$$

Accordingly, an alternative form of (11) is

$$
\begin{aligned}
e^{-a t} u(x, t)= & \frac{2 e^{-a t}}{\pi} \int_{0}^{\infty} \int_{0}^{\infty} e^{-\alpha \beta^{2} t} f(\xi) \sin \beta x \sin \beta \xi d \beta d \xi \\
& +\frac{x}{2 \sqrt{\pi \alpha}} \int_{0}^{t} e^{-a \eta} \varphi(t-\eta) e^{-x^{2} / 4 \alpha \eta} \eta^{-3 / 2} d \eta
\end{aligned}
$$

We proceed to the solution of the system (8), (9), (10). The Laplace transform $v^{*}(x, p)$ of the function $v(x, t)$ must satisfy the equations

$$
\frac{\partial^{2} v^{*}}{\partial x^{2}}-\frac{p+a}{\alpha} v^{*}=-\frac{b}{p \alpha}, \quad(12) ; \quad v^{*}(0, p)=0 .
$$

The solution of the system (12) and (13) is

$$
v^{*}(x, p)=\frac{b}{p(p+a)}\left(1-e^{-x \sqrt{(p+a) / \alpha})}=\sigma^{*}(p)-\sigma^{*}(p) w^{*}(p)\right.
$$

${ }^{2}$ H. S. Carslaw, Mathematical theory of the conduction of heat in solids, Macmillan and Co., London, $1921, \S 81$, p. 172. 
whence, by a well-known theorem, ${ }^{3}$

From

$$
v(x, t)=\sigma(t)-\int_{0}^{t} \sigma(t-\eta) w(\eta) d \eta .
$$

$$
\int_{0}^{\infty} e^{-p t} \sigma(t) d t=\sigma^{*}(p)=\frac{b}{p(p+a)}=\frac{b}{a}\left(\frac{1}{p}-\frac{1}{p+a}\right),
$$

it follows that

$$
\sigma(t)=\frac{b}{a}\left(1-e^{-a t}\right)
$$

If in the known identity

$$
\int_{0}^{\infty} e^{-p t} \sqrt{\frac{\lambda}{\pi}} e^{-\lambda / t} t^{-3 / 2} d t=e^{-2 \sqrt{\lambda p}}
$$

we put $\lambda=x^{2} / 4 \alpha$ and replace $p$ by $p+a$, we obtain

whence

$$
\frac{x}{2 \sqrt{\pi \alpha}} \int_{0}^{\infty} e^{-p t} e^{-a t} e^{-x^{2} / 4 \alpha t} t^{-3 / 2} d t=e^{-x \sqrt{(p+a) / \alpha}},
$$

$$
w(x, t)=\frac{x}{2 \sqrt{\pi \alpha}} e^{-a t} e^{-x^{2} / 4 \alpha t} t^{-3 / 2} .
$$

In view of (16) and (17), (15) becomes

$$
v(x, t)=-\frac{b}{2 a \sqrt{\pi \alpha}} \int_{0}^{t}\left\{1-e^{-a(t-\eta)}\right\} e^{-a \eta} e^{-x^{2} / 4 \alpha \eta} \eta^{-3 / 2} d \eta+\frac{b}{a}\left(1-e^{-a t}\right) .
$$

Making use of the identity

$$
\begin{aligned}
\int_{c}^{\infty} e^{-\left(a^{2 \lambda 4}+b^{2}\right) / \lambda^{2}} d \lambda= & \frac{\sqrt{\pi}}{2 a} \cosh 2 a b \\
& +\frac{\sqrt{\pi}}{4 a} e^{-2 a b} \operatorname{Erf}\left(\frac{b}{c}-a c\right)-\frac{\sqrt{\pi}}{4 a} e^{2 a b} \operatorname{Erf}\left(\frac{b}{c}+a c\right)
\end{aligned}
$$

and some elementary transformations, we may write (18) in the alternative form

$$
\begin{aligned}
& v(x, t)=\frac{b}{a}\left\{1-e^{-a t} \operatorname{Erf}\left(\frac{x}{2 \sqrt{\alpha t}}\right)\right\} \\
&-\frac{b}{2 a}\left\{2 \cosh x \sqrt{\frac{a}{\alpha}}+e^{-x \sqrt{a / \alpha}} \operatorname{Erf}\left(\sqrt{a t}-\frac{x}{2 \sqrt{\alpha t}}\right)\right. \\
&\left.-e^{x \sqrt{a / \alpha}} \operatorname{Erf}\left(\sqrt{a t}+\frac{x}{2 \sqrt{\alpha t}}\right)\right\},
\end{aligned}
$$

${ }^{3}$ See, for example, J. R. Carson, Electric circuit theory and operational calculus, McGraw-Hill Book Co., New York, 1926, p. 41.

4 J. R. Carson, loc. cit., p. 39.

5 'This is a slight generalization of Churchill's formula (4), page 120. 
where

$$
\operatorname{Erf}(x)=\frac{2}{\sqrt{\pi}} \int_{0}^{x} e^{-\beta^{2}} d \beta .
$$

It should be noted that in $\left(18^{\prime}\right)$ the function $v(x, t)$ is expressed in terms of tabulated functions.

The final solution of our problem is given by (4) in conjunction with (11) and (18) or $\left(11^{\prime}\right)$ and $\left(18^{\prime}\right)$.

\section{THE SPHERICAL GYROCOMPASS*}

BY WALTER KOHN (University of Toronto)

In the existing literature on gyroscopes ${ }^{1}$ the theory of the gyrocompass is developed for the case of a rotor whose ellipsoid of inertia is an ellipsoid of revolution. The mathematics of this treatment is somewhat involved and, in deducing the differential equations of motion, approximations based on the smallness of the earth's angular velocity are made. In the present communication we shall treat a gyrocompass the rotor of which has a spherical ellipsoid of inertia. The motion of such a gyrocompass is, of course, covered by the more general theory usually given, but owing to the symmetry of the sphere this case allows a considerably simpler, separate treatment in which, moreover, no approximations are necessary. At the same time the essential features of gryoscopic motion are preserved.

The following system will serve as a simple model of a spherical gyrocompass. The rotor is a rigid homogeneous sphere rotating freely about a light axle which passes through its centre. The ends of this axle can slide in a smooth horizontal ring which is concentric with the rotor and rigidly attached to the earth. When the rotor is set in rapid revolution about its axle the latter executes oscillations about the meridian which will now be examined.

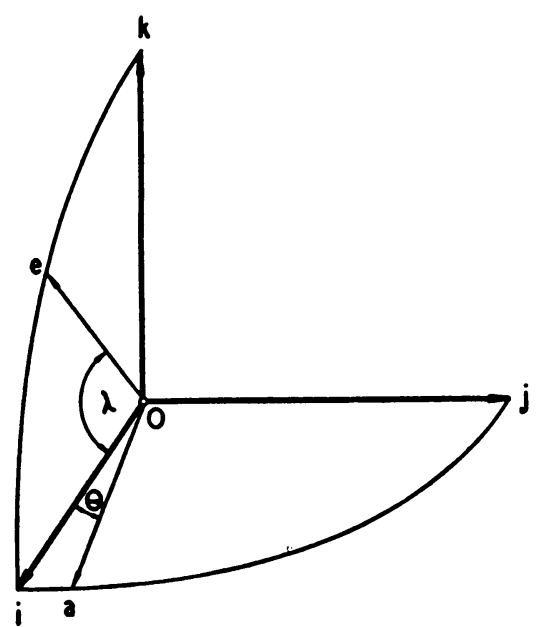

FIG. 1.

In the figure the right-handed unit triad, $\mathbf{i}, \mathbf{j}, \mathbf{k}$, which is fixed relative to the earth is defined as follows: $O$ is the center of the rotor; $\mathbf{k}$ lies in the direction of the upward vertical; $i$ lies along the meridian and points north; $\mathbf{j}$, pointing west, completes the triad. The unit vector, $\mathbf{a}$, lies along the axle of the gyrocompass and the unit vector, $\mathbf{e}$ (in the $\mathbf{i}, \mathbf{k}$ plane), is parallel to the earths' axis; thus the angle $\lambda$, between $i$ and $e$, is the latitude of the observer.

* Received July 10, 1944.

${ }^{1}$ Cf. T. Levi Civita and U. Amaldi, Lezioni di meccanica razionale, vol. 2, Zanichelli, Bologna, 1927, pp. 191-195; or J. L. Synge and B. A. Griffith, Principles of mechanics, McGraw-Hill Book Co., New York, 1942, pp. 430-433. 